\title{
Uso de redes neurais para predição da maturação do amendoim com imagens de UAV
}

\author{
Submetido - 04 jul. 2021 \\ Aprovado - 31 jul. 2021 \\ Publicado - \\ set. 2021
}

http://dx.doi.org/10.52755/sas.v.2i(edesp2)129

Jarlyson Brunno Costa Souza iD

Doutorando no programa de Pós-graduação em Produção Vegetal pela Universidade Estadual Paulista "Júlio de Mesquita Filho". E-mail: jarlyson.brunno@unesp.br

Samira Luns Hatum de Almeida iD

Doutorando no programa de Pós-graduação em Produção Vegetal pela Universidade Estadual Paulista "Júlio de Mesquita Filho. E-mail: samira.Ih.almeida.unesp.br.

\section{Armando Lopes de Brito de Filho (iD}

Doutorando no programa de Pós-graduação em Ciência do Solo pela Universidade Estadual Paulista "Júlio de Mesquita Filho". E-mail: armando.brito@unesp.br.

Graduando em Engenharia Agrícola pela Universidade Federal do Maranhã. E-mail: mirlas2@hotmail.com

Graduando em Agronomia leonardoagronomo@hotmail.com

pela

Universidade

Leonardo Barbosa Silva(iD

Federal do Maranhão. E-mail: Prof. Dr. Livre-Docente da UNESP/FCAV (Produção Vegetal), Jaboticabal-SP. E-mail: rouverson.silva@unesp.br.

\section{RESUMO}

Técnicas de sensoriamento remoto e o aprendizado de máquinas são ferramentas importantes para o setor agropecuário, e podem trazer melhorias significativa no gerenciamento agrícola. Diante disto, objetivou-se criar um método de predição da maturação do amendoim a partir de imagens de veículo aéreo não tripulado (UAV), utilizando redes neurais artificias (RNA). 0 experimento foi conduzido em talhão comercial na safra do ano 2019/20 no município de Dumont - SP. A coleta das imagens foi obtida através da câmera multiespectral Micasense RedEdge. Dois modelos de RNA's (RBF e MLP) foram utilizados para predizer o índice de maturação do amendoim, com as bandas espectrais e 7 índices de vegetação sendo utilizados na camada de entrada. Para validação dos modelos, foram utilizados $20 \%$ dos dados e para treinamento $80 \%$. O NDRE foi capaz de predizer o PMI com precisão (0.90 e 0.88 ) e acurácia (0.06 e 0.06) para as redes MLP e RBF respectivamente. A avaliação de desempenho dos modelos indica que as redes RBF e MLP são semelhantes para predizer a maturação do amendoim. Conclui-se com este trabalho que o índice de maturação pode ser previsto utilizando índices de vegetação a partir de imagens de UAV.

Palavras-chave: Agricultura digital; Inteligência Artificial; Sensoriamento remoto.

\section{Using neural networks for peanut maturity prediction with UAV images}

\section{ABSTRACT}

Remote sensing techniques and machine learning are important tools for the agricultural sector, and can bring significant improvements in agricultural management. In view of this, the

Este é um trabalho de acesso aberto e distribuído sob os Termos da Creative

Commons Attribution Attribution-NonCommercial-ShareAlike 4.0 International. 
objective was to create a method to predict peanut maturity from unmanned aerial vehicle (UAV) images, using artificial neural networks (ANN). The experiment was conducted in a commercial field in the 2019/20 crop year in the municipality of Dumont - SP. The collection of images was obtained through the Micasense RedEdge multispectral camera. Two ANN models (RBF and MLP) were used to predict the peanut maturity index, with the spectral bands and 7 vegetation indices being used in the input layer. For validation of the models, $20 \%$ of the data were used and for training $80 \%$. The NDRE was able to predict the PMI with accuracy $(0.90$ and 0.88) and precision (0.06 and 0.06) for the MLP and RBF networks respectively. The performance evaluation of the models indicates that the RBF and MLP networks are similar for predicting peanut maturity. It is concluded from this work that maturity index can be predicted using vegetation indices from UAV images.

Keywords: Digital Agriculture; Artificial Intelligence; Remote Sensing.

\section{Uso de redes neuronales para la predicción de la madurez} del amendoim con imágenes de UAV

\section{RESUMEN}

Las técnicas de teledetección y el aprendizaje automático son herramientas importantes para el sector agrícola, y pueden aportar mejoras significativas en la gestión agrícola. Por ello, se ha querido crear un método para predecir la madurez del cacahuete a partir de imágenes de vehículos aéreos no tripulados (UAV), utilizando redes neuronales artificiales (RNA). EI experimento se realizó en un rodal comercial en la campaña 2019/20 en el municipio de Dumont - SP. La colección de imágenes se obtuvo mediante la cámara multiespectral Micasense RedEdge. Se utilizaron dos modelos de RNA (RBF y MLP) para predecir el índice de madurez del cacahuete, utilizando las bandas espectrales y los 7 índices de vegetación en la capa de entrada. Para la validación de los modelos se utilizó el $20 \%$ de los datos y para el entrenamiento el $80 \%$. EI NDRE fue capaz de predecir el PMI con una exactitud $(0,90$ y 0,88) y precisión $(0,06$ y 0,06) para las redes MLP y RBF respectivamente. La evaluación del rendimiento de los modelos indica que las redes $R B F$ y MLP son similares para predecir la madurez del cacahuete. De este trabajo se concluye que el índice de madurez puede predecirse utilizando índices de vegetación a partir de imágenes de UAV.

Palabras clave: Agricultura digital; inteligencia artificial; teledetección.

\section{Introdução}

O sensoriamento remoto e o aprendizado de máquinas são duas importantes ferramentas para o setor agropecuário, e podem contribuir de forma significativa com o gerenciamento dos recursos e o processo de tomada de decisão. Isso é possível devido à grande disponibilidade de dados obtidos a partir destas tecnologias, que quando transformadas em informação são de grande relevância para o monitoramento agrícola (KATTENBORN et al., 2020).

$\mathrm{Na}$ cultura do amendoim, um dos gargalos é a dificuldade na definição do momento ideal do arranquio baseada na maturação das vagens, uma vez que interfere diretamente na quantidade e qualidade do 
produto e consequentemente no retorno financeiro ao produtor (ROWLAND et al., 2006). Diante da importância da maturação do amendoim, diversos métodos foram criados ao longo dos anos. No entanto, a metodologia mais difundida e aceita pelos produtores de amendoim, o quadro de maturação de Williams e Drexler (1981) é extremamente subjetiva. Além disso, a alta variabilidade presente no campo exige a coleta de muitas amostras destrutivas de amendoim, o que é trabalhoso e prejudica a produção final (ASHAPURE et al., 2019).

Visando desenvolver método mais eficiente e preciso para determinar o PMI (Peanuty Maturity Index) do amendoim, pesquisas envolvendo tecnologias de Redes Neurais Artificias (RNA's) e Sensoriamento Remoto (SR) podem revolucionar o setor. O uso do SR oferece alternativas com sensores embarcados em UAV (Unmanned Aerial Vahicle), que permite o estudo da vegetação com alta resolução espacial por meio de técnicas (índices de vegetação) que são sensíveis às propriedades fisiológicas e morfológicas das plantas (KATTENBORN et al., 2021). Logo, o uso de plataformas de SR com o auxílio de RNA's podem criar modelos capazes de prever o PMI do amendoim de forma precisa e acurada.

Com base nisso, objetivou-se com este trabalho avaliar o potencial do UAV para predizer a maturação do amendoim, utilizando técnicas de aprendizado de máquinas; além de validar o desempenho de predição da maturação utilizando bandas espectrais e índices de vegetação com dois tipos de RNA's: a perceptron multicamadas (MLP) e a função de ativação de base radial (RBF).

\section{Material e métodos}

O experimento foi conduzido em talhão comercial na safra do ano 2019/20 no município de Dumont - SP. A cultivar utilizada foi a IAC OL3 tipo Runner. O solo da área experimental possui textura argilosa, sendo classificado como LATOSSOLO VERMELHO de acordo com Embrapa (2013). O experimento foi realizado em área de aproximadamente $8 \mathrm{ha}$, com 
30 pontos equidistantes a 50 metros. Realizou-se avaliações da maturação do amendoim aos 75, 105, 120 e 125 dias após a semeadura (DAS).

Para a análise de maturação utilizou-se o método Peanut Maturity Index (PMI), que calcula a porcentagem de vagens maduras para cada amostra (Rowland et al. 2006). Foram retiradas todas as vagens das plantas contidas na amostra (5 plantas por ponto), obtendo-se em média 150 a 250 vagens para análise de maturação em cada ponto amostral. Em seguida, todas a vagens foram submetidas ao processo de remoção do exocarpo com o auxílio de lavadora de alta pressão em laboratório para expor a coloração das vagens (WILLIAMS E DREXLER, 1981).

Para obtenção das imagens aéreas foi realizado voos com aeronave remotamente pilota (UAV) modelo DJI Matrice 100 (Shenzhen, China) com a câmera Micasense RedEdge-M, que captura imagens em 5 bandas espectrais: Azul (465-485nm), Verde (550-570 nm), Vermelho (658-678 nm), Infravermelho $(820-860 \mathrm{~nm})$ e Red Edge $(712-722 \mathrm{~nm})$. Os voos foram realizados à $90 \mathrm{~m}$ de altitudes com sobreposição lateral e frontal de $90 \%$. Para esta altura de voo a resolução espacial da câmera foi de $7,96 \mathrm{~cm}$.

Para fazer o processamento das imagens foi utilizado o software Pix4D, versão Student, que foi aplicado para gerar os ortomosaicos e a correção radiométrica das imagens. Foi realizada a extração do solo nas imagens por meio de classificação não supervisionada, a fim de evitar influências da reflectância do solo nas informações geradas pelo alvo (Planta). O software utilizado foi o Qgis (QGIS Development Team, OpenSource Geospatial Foundation, Chicago, IL, EUA).

A partir dos valores de refletância extraídos dos ortomosaicos para as imagens aéreas, foram calculados os seguintes índices de vegetação: NDVI, GNDVI, MNLI, EVI, NLI, SAVI e NDRE. Todos esses índices foram escolhidos por apresentarem grande aplicação na previsão de parâmetros agronômicos em diversas culturas.

Dois modelos de RNA's foram utilizados para predizer o índice de maturação do amendoim. As arquiteturas utilizadas foram do tipo função de 
ativação de base radial (RBF) e perceptron de multicamadas (MLP). As camadas de entrada para as duas redes (RBF e MLP), foram compostas pelos índices de vegetação e bandas espectrais e a camada de saída pelo PMI. O número de neurônios variou de 1 a 20 para as redes. Foram classificados 10 modelos (5 RBF e 5 MLP) de 1000 testados

Para validação e treinamento dos modelos, o banco de dados foi divido em $80 \%$ para treinamento e $20 \%$ para validação. Os procedimentos de treinamento e validação dos modelos das redes neurais foram implementados no pacote Neural Networks do software de análise de dados Statistica (Statistica 7.0, Statsoft Inc., Tulsa, OK).

A eficiência das redes foi analisada por meio de gráficos com a relação 1:1 (SOARES et al., 2014). A acurácia foi analisada a partir do erro absoluto (MAE) e o coeficiente de determinação (R2) para precisão, demonstrando por meio destes cálculos a confiabilidade dos dados obtidos para prever o $\mathrm{PMI}$, e ainda, foram realizadas análises comparativas entre os dados preditos com os reais.

\section{Resultados e discussão}

Dentre os resultados obtidos, considerou-se a melhor arquitetura de rede aquelas que apresentaram acurácia (MAE) entre 0.05 e 0.06 e precisão $\left(R^{2}\right)$ acima de 0.85 . Diante disto, apenas 4 modelos demonstraram potencial para predizer a maturação do amendoim (Figura 1). A rede RBF utilizou 3 entradas para o modelo com índices de vegetação, enquanto o modelo com bandas espectrais e índices de vegetação para a rede MLP, utilizou 5 entradas. Logo, o perfil do modelo da rede MLP foi 5:5-11-10-1:1 e para rede RBF foi 3:3-20-1:1.

Para o modelo que utilizou somente IVs, a análise de sensitividade na Tabela 1, indicou o NDRE como sendo a variável mais importante da rede, seguido do NLI e SAVI. Já para a MLP que combinou índice com bandas, o NLI foi a variável mais importante seguido dos índices NDRE, MNLI, EVI e green. 
A rede utilizou apenas a banda do green para predizer a maturação que foi classificada como a quinta variável mais importante. A banda do green já demonstrou em diversos estudos, maior sensibilidade ao conteúdo de clorofila (clorofila a e b) e biomassa no monitoramento da produção do milho, soja, batata e trigo (SKAKUN et al., 2021; GITELSON et al., 1996; GITELSON et al., 2003; NGUY ROBERTSON et al., 2014; VINÃ et al., 2011).

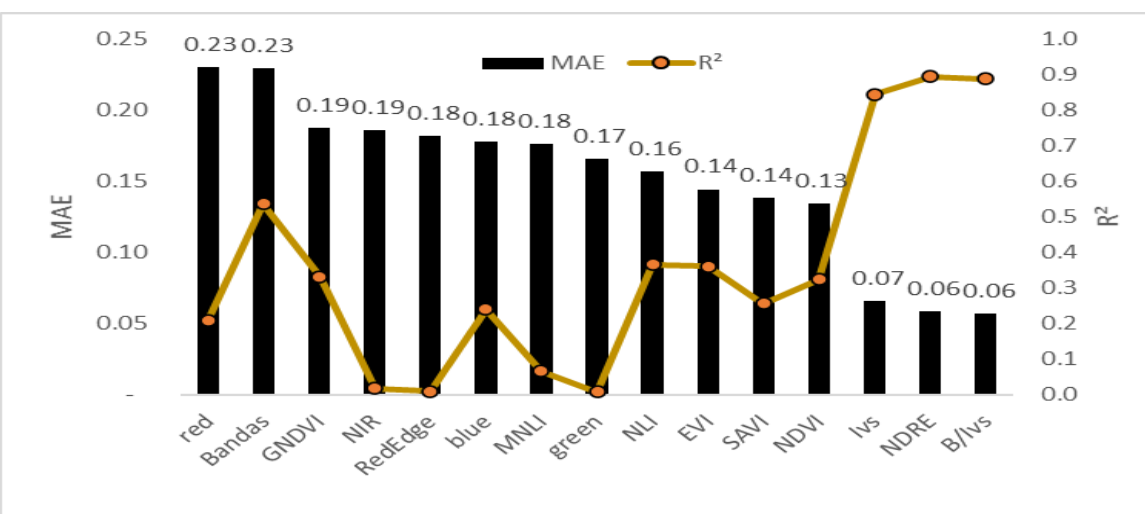

(A)

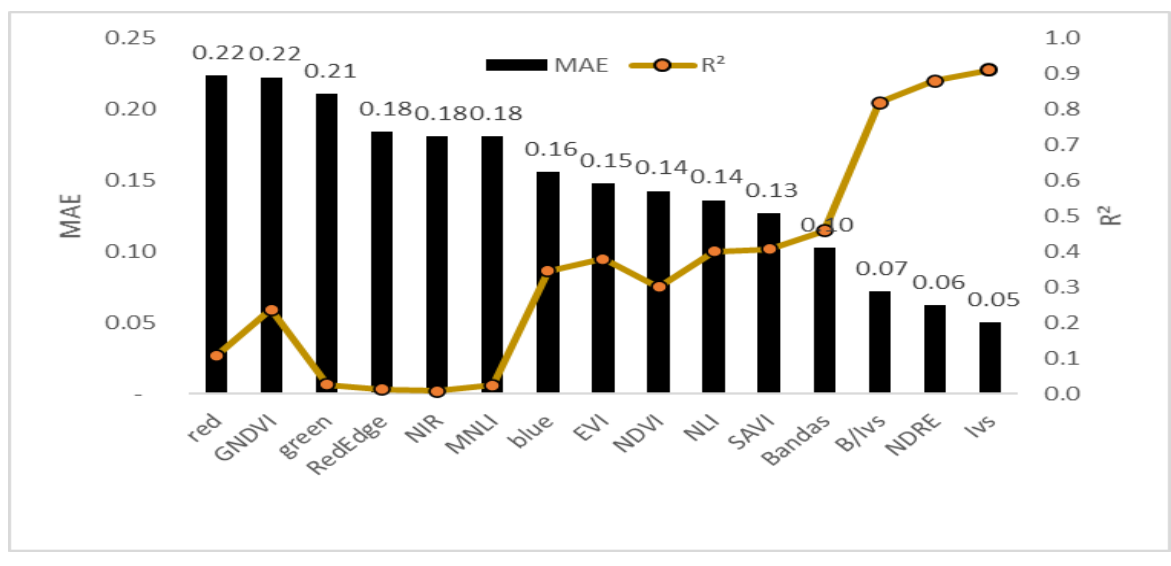

(B)

Figura 1. Acurácia (Mean Absolute Error) e precisão $\left(R^{2}\right)$ da validação dos modelos para as redes $\operatorname{MLP}(\mathrm{A}) \operatorname{RBF}(\mathrm{B})$.

Quando os modelos foram treinados e validados utilizando as variáveis separadamente, somente o NDRE foi capaz de predizer o PMI com precisão (0.90 e 0.88) e acurácia (0.06 e 0.06) para as redes MLP e RBF respectivamente (Figura 1). Este índice de vegetação já demonstrou em estudos anteriores, potencial para avaliar o comportamento de culturas com alta densidade de biomassa, pois a borda do vermelho (RedEdge - RE) o 
deixa menos sensível a estrutura do dossel da planta, o que torna o NDRE bastante eficiente para o estudo da vegetação (MORLIN, 2019). Estudos demonstraram que a banda RE tem alta correlação com diversos parâmetros fisiológicos da vegetação, como teor de nitrogênio, clorofila e biomassa (CLEVERS; GITELSON 2013). Outro fator que pode ter influenciado para os resultados com o NDRE é que a banda do RE também apresenta menos sensibilidade a ruídos espectrais que são gerados pela radiação e condições atmosféricas (CHO et al. 2008).

Tabela 1. Análise de sensitividade com nível de importância das variáveis de entrada para combinações das redes MLP e RBF.

\begin{tabular}{ccc}
\hline Bandas e IV's & \multicolumn{2}{c}{ Combinações } \\
\cline { 2 - 3 } & Bandas/IV's (MLP) & Índices de Vegetação (RBF) \\
\hline green & 5 & 1 \\
\hline NDRE & 2 & 2 \\
\hline NLI & 1 & \\
\hline MNLI & 3 & 3 \\
\hline EVI & 4 & \\
\hline NDVI & &
\end{tabular}

V's = Índices de vegetação; MLP = Perceptron Multicamadas; RBF = Função de base de ativação radial.

Na Figura 2 é demonstrada a avaliação de desempenho dos modelos RBF e MLP para o NDRE. O índice de maturação estimado pelo modelo resultante foi comparado com o PMI determinado pelo método Hull Scrap (método de campo). A avaliação de desempenho dos modelos indica que as redes RBF e MLP são semelhantes para predizer a maturação do amendoim com o NDRE. 


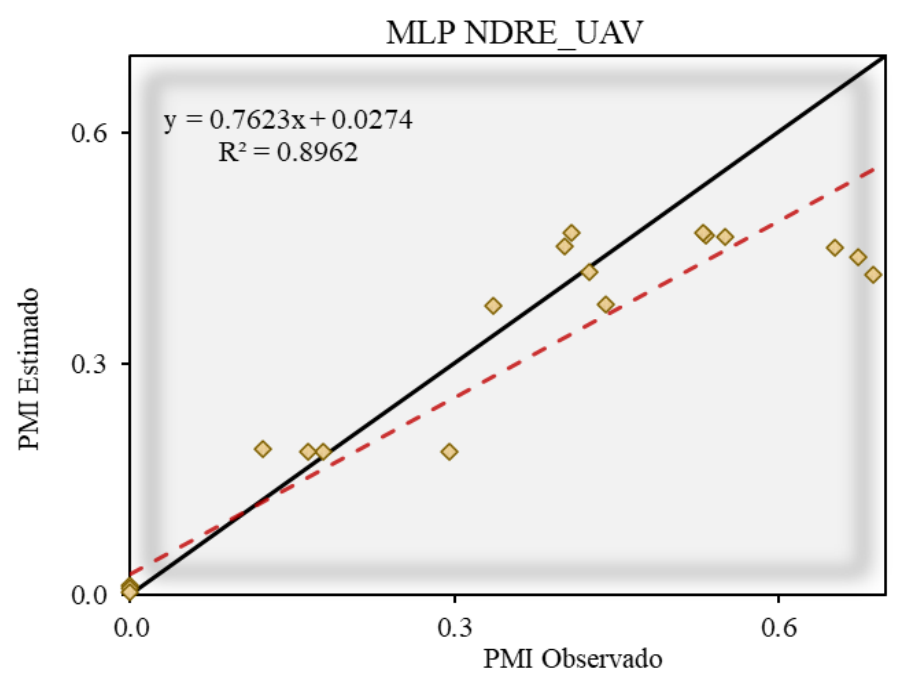

(A)

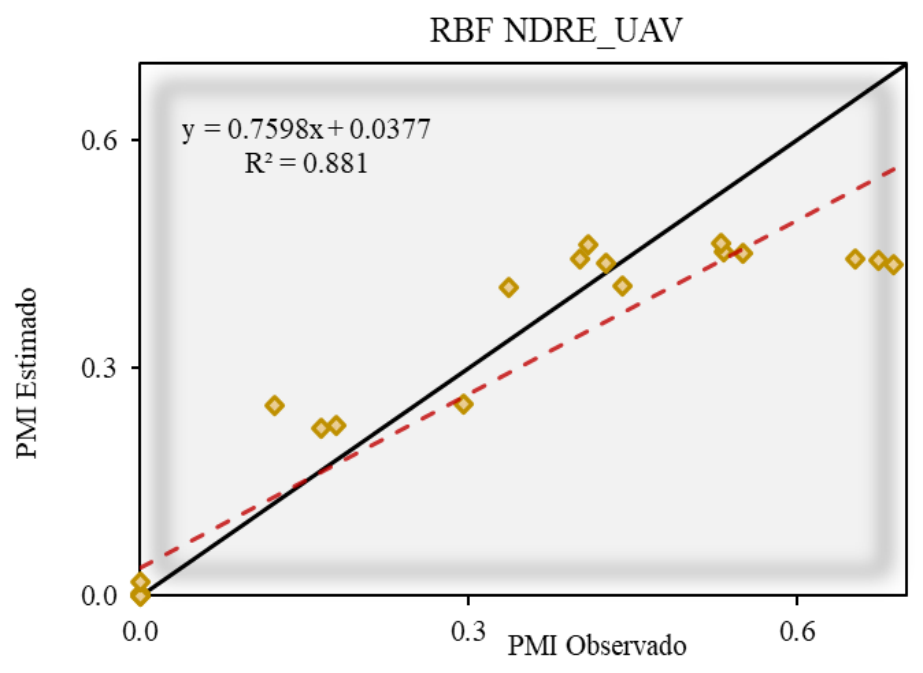

(B)

Figura 2. Desempenho dos modelos do NDRE para as redes neurais MLP (A) e RBF (B).

\section{Conclusões}

Este trabalho demonstrou o potencial uso de imagens de UAV para predizer a maturação do amendoim utilizando o índice de vegetação NDRE. Os modelos de redes MLP e RBF mostraram-se capazes de predizer o PMI com boa acurácia e precisão. Visando desenvolver métodos robustos, é importante a realização de estudos futuros que possam observar o comportamento das bandas espectrais e IV's em diferentes condições de cultivo e cultivares. Desta forma, seria possível desenvolver um software capaz de determinar o momento ideal de colheita com base em imagens de UAV. 


\section{Referências}

Ashapure, A., Jung, J., Chang, A., Oh, S., Maeda, M., \& Landivar, J. (2019). A comparative study of RGB and multispectral sensor-based cotton canopy cover modelling using multi-temporal UAS data. Remote Sensing, 11(23), 2757. https://doi.org/10.3390/rs11232757

Cho, MA, Skidmore, AK, \& Atzberger, C. (2008). Em direção a posições de borda vermelha menos sensíveis aos parâmetros biofísicos do dossel para estimativa de clorofila foliar usando dados simulados de propriedades optique Spectrales des feuilles (PROSPECT) e espalhamento por folhas inclinadas arbitrariamente (SAILH). International Journal of Remote Sensing, 29 (8), 2241-2255. https://doi.org/10.1080/01431160701395328

Clevers, J. G., \& Gitelson, A. A. (2013). Remote estimation of crop and grass chlorophyll and nitrogen content using red-edge bands on Sentinel-2 and-3. International Journal of Applied Earth Observation and Geoinformation, 23, 344-351. https://doi.org/10.1016/j.jag.2012.10.008

Embrapa-Empresa Brasileira de Pesquisa Agropecuária. (2013). Sistema Brasileiro de Classificação de Solos (3rd ed. Rev. ampl., p. 353). Brasília, DF: Empresa Brasileira de Pesquisa Agropecuária (Embrapa).

Gitelson, A. A., Viña, A., Arkebauer, T. J., Rundquist, D. C., Keydan, G., \& Leavitt, B. (2003). Remote estimation of leaf area index and green leaf biomass in maize canopies. Geophysical research letters, 30(5).

Gitelson, Anatoly A.; Kaufman, Yoram J.; Merzlyak, Mark N. (1996). Use of a green channel in remote sensing of global vegetation from EOS-MODIS. Remote sensing of Environment, v. 58, n. 3, p. 289-298, https://doi.org/10.1016/S0034-4257(96)00072-7

Kattenborn, T., Eichel, J., Wiser, S., Burrows, L., Fassnacht, F. E., \& Schmidtlein, S. (2020). Convolutional Neural Networks accurately predict cover fractions of plant species and communities in Unmanned Aerial Vehicle imagery. Remote Sensing in Ecology and Conservation, 6(4), 472-486. https://doi.org/10.1002/rse2.146

Kattenborn, T., Leitloff, J., Schiefer, F., \& Hinz, S. (2021). Review on Convolutional Neural Networks (CNN) in vegetation remote sensing. ISPRS Journal of Photogrammetry and Remote Sensing, 173, 24-49. https://doi.org/10.1016/j.isprsjprs.2020.12.010

Nguy-Robertson, A. L., Peng, Y., Gitelson, A. A., Arkebauer, T. J., Pimstein, A., Herrmann, I., ... \& Bonfil, D. J. (2014). Estimating green LAI in four crops: Potential of determining optimal spectral bands for a universal algorithm. Agricultural and forest meteorology, 192, 140-148. https://doi.org/10.1016/j.agrformet.2014.03.004

Rowland, D. L., Sorensen, R. B., Butts, C. L., \& Faircloth, W. H. (2006). Determination of maturity and degree day indices and their success in 
predicting peanut maturity. Peanut Science, 33, 125-136. https://doi.org/10.3146/0095-

Skakun, S., Kalecinski, NI., Brown, MGL., Johnson, DM., Vermote, EF., Roger, J.-C.; Franch, B. (2021) Avaliando a variação de rendimento de milho e soja no campo a partir de imagens de satélite WorldView-3, Planet, Sentinel-2 e Landsat 8. Remote Sens. 13, 872. https://doi.org/10.3390/rs13050872

Viña, A., Gitelson, A. A., Nguy-Robertson, A. L., \& Peng, Y. (2011). Comparison of different vegetation indices for the remote assessment of green leaf area index of crops. Remote Sensing of Environment, 115(12), 3468-3478. https://doi.org/10.1016/j.rse.2011.08.010

Williams, E. J., \& Drexler, J. S. (1981). A non-destructive method for determining peanut pod maturity. Peanut Science, 8(2), 134-141. https://doi.org/10.3146/i0095-3679-8-2-15 